\title{
THE COMPOSITION OF INDIAN COWS AND BUFFALOES' MILK.
}

\author{
By J. Walter Leather, Ph.D., F.I.C.
}

INFORMATION as to the composition of Indian cows and buffaloes' milk will doubtless be of interest to members of the Society of Public Analysts, and I submit now the following results of analyses made by myself. I may mention than an idea had become current in India that the milk of Indian cows is different in composition to that of European cows, and accordingly complete analyses were in each case made. As to the composition of buffaloes' milk, no reliable information existed previously.

In Table I. are given analyses of milking from a number of cows at the Poona Dairy Farm (Bombay Presidency), and in Table II. similar information regarding milk from cows of the Saidapet herd (Madras). Table III. exhibits the composition of buffaloes' milk (Poona herd).

Firstly, as to Indian cows' milk. It is found to differ in no essential particulars from that met with in Europe. The relations existing between the specific gravity, the solids-not-fat, and the fat agree well in all cases with those which Richmond and others have found in the case of milks from English cows. So also, as shown in Table IV., the relationship between the milk-sugar, the proteids, and the mineral matter agrees well with the proportions $13: 9: 2$. The percentage of butter-fat is high, varying from 4 to 6 . Indian cows' milk is invariably very nearly white and the butter very pale yellow, unless coloured artificially.

Secondly, as to buffaloes' milk. It will be seen that the majority of the samples analysed are characterized by an extraordinarily high proportion of butter-fat, 7 and 8 per cent. being common, and in one case close on 10 per cent. of fat was found. Buffaloes' milk is white and the butter is also usually quite white.

As will be seen from Table III., the relation existing between the specific gravity, the solids-not-fat, and the fat is the same as for cows' milk, the solids-notfat, when calculated by Richmond's formula, agreeing well with those found by analysis.

The percentage of proteids in buffaloes' milk is distinctly higher than in cows' milk, and varies from 3.5 to 4.3 per cent. The percentages of milk-sugar and mineral matter correspond very closely with those of cows' milk. On the other hand, the relationship between these three ingredients is not the same as for cows' milk. In one particular sample (that of the mixed milk of the herd) the percentage of proteids was higher than I found in most of the individual milks; but this does not materially alter the fact. If one takes the mean figures, the relationship works out to about $10: 12: 2$. It is probable that the proportion of proteids is necessarily higher in a milk containing so much butter-fat, in order that the albuminoid ratio may be maintained. 
Table I.-Composition of Indian Cows' Milk. Poona Herd (Bombay), 1899.

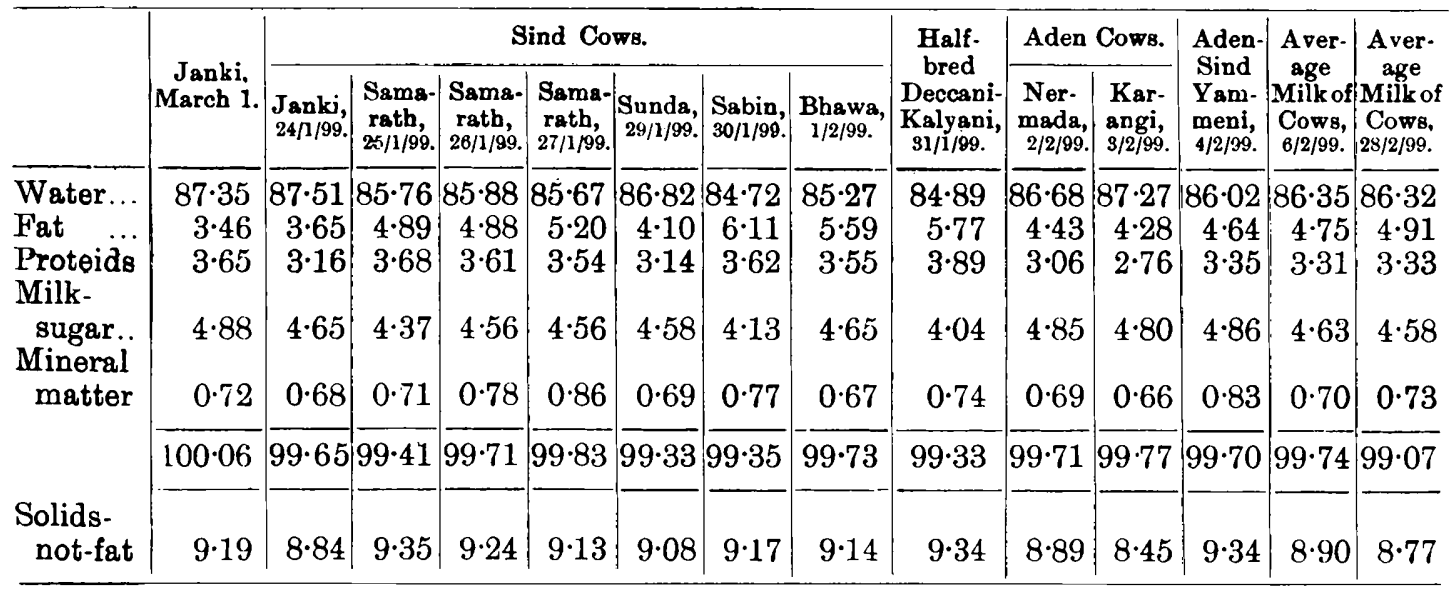

Table II.-Composition of Cows' Milk. Saidapet Herd (Madras), 1900.

\begin{tabular}{|c|c|c|c|c|c|c|c|c|c|}
\hline & $\begin{array}{c}\text { Average } \\
\text { Milk of } \\
\text { Herd, } \\
29 / 3 / 00 \text {. }\end{array}$ & $\begin{array}{c}\text { A verage } \\
\text { Milk of } \\
\text { Herd, } \\
4 / 4 / 00 .\end{array}$ & $\begin{array}{c}\text { Average } \\
\text { Milk of } \\
\text { Herd, } \\
\tau / 4 / 00\end{array}$ & $\begin{array}{c}\text { Baggeon } \\
\text { II., } \\
28 / 3 / 00 .\end{array}$ & $\begin{array}{c}\text { Govindi, } \\
2 / 4 / 00\end{array}$ & $\begin{array}{l}\text { Parvati, } \\
3 / 4 / 00 .\end{array}$ & $\begin{array}{l}\text { Manachu } \\
\text { II., } \\
5 / 4 / 00 .\end{array}$ & $\begin{array}{c}\text { Poongan, } \\
6 / 4 / 00\end{array}$ & $\begin{array}{l}\text { Manachu } \\
\text { III., } \\
9 / 4 / 00 .\end{array}$ \\
\hline $\begin{array}{lll}\text { Water } & \ldots & \ldots \\
\text { Fat } & \ldots & \ldots \\
\text { Proteids } \quad \ldots & \ldots \\
\text { Milk-sugar } & \ldots \\
\text { Mineral matter } & \ldots\end{array}$ & $\begin{array}{r}86 \cdot 27 \\
4 \cdot 00 \\
3 \cdot 15 \\
4 \cdot 82 \\
0 \cdot 76\end{array}$ & $\begin{array}{r}86 \cdot 87 \\
4 \cdot 14 \\
3 \cdot 16 \\
4 \cdot 81 \\
0 \cdot 74\end{array}$ & $\begin{array}{r}85 \cdot 97 \\
4 \cdot 68 \\
3 \cdot 23 \\
4 \cdot 92 \\
0 \cdot 72\end{array}$ & $\begin{array}{r}86 \cdot 14 \\
4 \cdot 41 \\
3 \cdot 23 \\
5 \cdot 03 \\
0 \cdot 76\end{array}$ & $\begin{array}{r}85 \cdot 52 \\
5 \cdot 48 \\
3 \cdot 14 \\
4 \cdot 68 \\
0 \cdot 70\end{array}$ & $\begin{array}{r}84 \cdot 48 \\
6 \cdot 07 \\
3 \cdot 27 \\
4 \cdot 66 \\
0 \cdot 72\end{array}$ & $\begin{array}{r}86 \cdot 13 \\
4 \cdot 57 \\
3 \cdot 44 \\
4 \cdot 56 \\
0 \cdot 77\end{array}$ & $\begin{array}{r}86 \cdot 98 \\
3 \cdot 52 \\
3 \cdot 27 \\
4 \cdot 96 \\
0 \cdot 75\end{array}$ & $\begin{array}{r}86 \cdot 08 \\
4 \cdot 62 \\
3 \cdot 25 \\
5 \cdot 05 \\
0 \cdot 77\end{array}$ \\
\hline & $99 \cdot 00$ & $99 \cdot 72$ & $99 \cdot 52$ & $99 \cdot 59$ & $99 \cdot 52$ & $99 \cdot 20$ & $99 \cdot 47$ & $99 \cdot 48$ & $99 \cdot 77$ \\
\hline $\begin{array}{l}\text { Solids-not-fat } \ldots \\
\text { Specific gravity at } \\
15 \cdot 5^{\circ} \text { C. . . } \ldots \\
\text { Solids-not-fat calcu- } \\
\text { lated by Rich- } \\
\text { mond's formula }\end{array}$ & $\begin{array}{r}9 \cdot 73 \\
1033 \cdot 50\end{array}$ & $\begin{array}{r}8.99 \\
1032 \cdot 0\end{array}$ & $\begin{array}{r}9 \cdot 35 \\
1032 \cdot 0\end{array}$ & $\begin{array}{r}9 \cdot 45 \\
1033 \cdot 0\end{array}$ & $1031 \cdot 0$ & $1033 \cdot 0$ & 1032.0 & $1034 \cdot 0$ & $1033 \cdot 0$ \\
\hline
\end{tabular}

Table III.-Indian Buffaloes' Milk. Poona Herd (Bombay).

\begin{tabular}{|c|c|c|c|c|c|c|c|c|}
\hline & & \multicolumn{4}{|c|}{ Jaffarabadis. } & \multicolumn{3}{|c|}{ Suratis. } \\
\hline & & $\begin{array}{l}\text { Nevasi, } \\
6 / 2 / 99\end{array}$ & $\begin{array}{l}\text { Nevasi, } \\
13 / 2 / 99 \text {. }\end{array}$ & $\begin{array}{l}\text { Champa, } \\
7 / 2 / 99 .\end{array}$ & $\begin{array}{c}\text { Thami, } \\
\text { 10/2/99. }\end{array}$ & $\begin{array}{c}\text { Kevada, } \\
8 / 2 / 99 .\end{array}$ & $\begin{array}{l}\text { Phonga, } \\
\text { 9/2/99. }\end{array}$ & $\begin{array}{l}\text { Mangel, } \\
11 / 2 / 99 .\end{array}$ \\
\hline $\begin{array}{lr}\text { Water } & \ldots \\
\text { Fat .. } & \ldots \\
\text { Proteids } & \ldots \\
\text { Milk-sugar } & \ldots \\
\text { Mineral matter }\end{array}$ & $\begin{array}{l}\ldots \\
\ldots \\
\cdots \\
\cdots \\
\cdots\end{array}$ & $\begin{array}{r}86 \cdot 43 \\
4 \cdot 08 \\
3 \cdot 29 \\
5 \cdot 12 \\
0 \cdot 80\end{array}$ & $\begin{array}{r}85 \cdot 09 \\
5 \cdot 78 \\
3 \cdot 24 \\
5 \cdot 09 \\
0 \cdot 68\end{array}$ & $\begin{array}{r}82 \cdot 84 \\
7 \cdot 32 \\
4 \cdot 36 \\
4 \cdot 42 \\
0 \cdot 75\end{array}$ & $\begin{array}{r}84 \cdot 05 \\
6 \cdot 89 \\
3 \cdot 53 \\
4 \cdot 70 \\
0 \cdot 82\end{array}$ & $\begin{array}{r}82 \cdot 63 \\
7 \cdot 74 \\
3 \cdot 75 \\
4 \cdot 94 \\
0 \cdot 68\end{array}$ & $\begin{array}{r}83 \cdot 38 \\
6 \cdot 86 \\
3 \cdot 99 \\
4 \cdot 70 \\
0 \cdot 72\end{array}$ & $\begin{array}{r}81 \cdot 74 \\
8 \cdot 66 \\
4 \cdot 32 \\
4 \cdot 66 \\
0 \cdot 80\end{array}$ \\
\hline Mineral matter & & $99 \cdot 72$ & $99 \cdot 88$ & $99 \cdot 69$ & $99 \cdot 99$ & $99 \cdot 74$ & $99 \cdot 65$ & $100 \cdot 18$ \\
\hline Solids-not-fat & ... & $9 \cdot 49$ & $9 \cdot 13$ & $9 \cdot 06$ & $9 \cdot 63$ & $9 \cdot 63$ & $9 \cdot 76$ & $9 \cdot 60$ \\
\hline
\end{tabular}


THE ANALYST.

TABLE IV.

\begin{tabular}{|c|c|c|c|c|c|c|c|c|c|c|c|}
\hline & $\begin{array}{l}\text { Nevasi, } \\
28 / 2 / 99 .\end{array}$ & $\begin{array}{l}\text { Gaura, } \\
2 / 3 / 99 .\end{array}$ & $\begin{array}{c}\text { Jama- } \\
\text { dar, } \\
3 / 3 / 99 .\end{array}$ & $\begin{array}{l}\text { Kaoli, } \\
\text { 3/3/99. }\end{array}$ & $\begin{array}{l}\text { Bhadra, } \\
4 / 3 / 99 .\end{array}$ & $\begin{array}{l}\text { Asman, } \\
\text { 4/3/99. }\end{array}$ & $\begin{array}{l}\text { Gulab, } \\
6 / 3 / 99 .\end{array}$ & $\begin{array}{l}\text { Mixed } \\
\text { Milk of } \\
\text { Kirkee } \\
\text { Buffa- } \\
\text { loes. }\end{array}$ & $\begin{array}{c}\text { Rekhmi, } \\
6 / 3 / 99\end{array}$ & $\begin{array}{l}\text { Avadi, } \\
7 / 3 / 99\end{array}$ & $\begin{array}{l}\text { Chandi, } \\
7 / 3 / 99 .\end{array}$ \\
\hline $\begin{array}{ll}\text { Water ... } & \ldots \\
\text { Fat } \ldots & \ldots \\
\text { Proteids } & \ldots \\
\text { Milk-sugar } & \ldots \\
\text { Mineral matter }\end{array}$ & $\begin{array}{r}85 \cdot 78 \\
5 \cdot 17 \\
3 \cdot 31 \\
4 \cdot 97 \\
0 \cdot 70\end{array}$ & $\begin{array}{r}82 \cdot 16 \\
8 \cdot 14 \\
4 \cdot 33 \\
4 \cdot 27 \\
0 \cdot 77\end{array}$ & $\begin{array}{r}80 \cdot 16 \\
9 \cdot 95 \\
4 \cdot 04 \\
4 \cdot 58 \\
0 \cdot 79\end{array}$ & $\begin{array}{r}81 \cdot 57 \\
8 \cdot 79 \\
3 \cdot 86 \\
4 \cdot 66 \\
0 \cdot 71\end{array}$ & $\begin{array}{r}82 \cdot 89 \\
7 \cdot 56 \\
3 \cdot 62 \\
4 \cdot 87 \\
0 \cdot 71\end{array}$ & $\begin{array}{r}81 \cdot 15 \\
8 \cdot 81 \\
4 \cdot 31 \\
4 \cdot 61 \\
0 \cdot 76\end{array}$ & $\begin{array}{r}82 \cdot 63 \\
7 \cdot 59 \\
4 \cdot 39 \\
4 \cdot 89 \\
0 \cdot 73\end{array}$ & $\begin{array}{r}82 \cdot 22 \\
8 \cdot 09 \\
4 \cdot 34 \\
4 \cdot 56 \\
0 \cdot 76\end{array}$ & $\begin{array}{r}82 \cdot 74 \\
7 \cdot 42 \\
3 \cdot 91 \\
4 \cdot 65 \\
0 \cdot 81\end{array}$ & $\begin{array}{r}82 \cdot 52 \\
7 \cdot 52 \\
3 \cdot 89 \\
4 \cdot 60 \\
0 \cdot 80\end{array}$ & $\begin{array}{r}83 \cdot 23 \\
6 \cdot 95 \\
3 \cdot 89 \\
4 \cdot 67 \\
0 \cdot 75\end{array}$ \\
\hline & $99 \cdot 93$ & $99 \cdot 67$ & $99 \cdot 52$ & $99 \cdot 59$ & $99 \cdot 65$ & $99 \cdot 64$ & $100 \cdot 23$ & 99.97 & 99.53 & $99 \cdot 33$ & $99 \cdot 49$ \\
\hline Solids-not-fat ... & $9 \cdot 05$ & $9 \cdot 70$ & $9 \cdot 89$ & $9 \cdot 64$ & $9 \cdot 55$ & $10 \cdot 04$ & $9 \cdot 78$ & $9 \cdot 69$ & $9 \cdot 84$ & $9 \cdot 96$ & $9 \cdot 84$ \\
\hline $\begin{array}{l}\text { at } 15 \cdot 5^{\circ} \mathrm{C} . \\
\text { Solids-not-fat, } \\
\text { calculated by } \\
\text { Richmond's } \\
\text { formula } \quad . .\end{array}$ & $1034 \cdot 5$ & $1032 \cdot 1$ & $1031 \cdot 2$ & $1032 \cdot 0$ & $1032 \cdot 9$ & $1032 \cdot 9$ & $1032 \cdot 7$ & $1032 \cdot 1$ & $1034: 3$ & $1033 \cdot 8$ & 1033.9 \\
\hline
\end{tabular}

